REVIEW ARTICLE

\title{
Bacteriostatic preserved saline for pain-free periocular
} injections: review

\author{
Samantha Vicki Hunt $\mathbb{D}^{1 凶}$ and Raman Malhotra ${ }^{1}$ \\ (c) The Author(s), under exclusive licence to The Royal College of Ophthalmologists 2022
}

\begin{abstract}
We review evidence regarding the use of $0.9 \%$ benzyl alcohol in $0.9 \%$ sodium chloride solution for periocular injections ('preserved saline') including botulinum toxin A injections and local anaesthesia. A literature search was undertaken using search terms 'bacteriostatic saline', 'benzyl-alcohol saline', 'benzyl alcohol sodium chloride' and 'preserved saline'. Bibliographies identified further sources. There have been 62 studies published on the subject of preserved-saline since 1928. Significantly lower injectionassociated pain levels for periocular/facial botulinum toxin injections reconstituted with preserved-saline rather than preservativefree saline are reported by 5 studies. Significantly lower injection-associated pain with preserved-saline diluted lidocaine and epinephrine solution for eyelid anaesthesia compared with unmodified or buffered lidocaine with epinephrine, and adequate anaesthesia, was reported by one study. Thirty-one studies have explored preserved-saline for anaesthetic and seven for bacteriostatic properties, with very low infection rates after periocular botulinum toxin injections, and reduced rates of infection in indwelling catheters when preserved saline is used to flush. A meta-analysis concluded that lidocaine-containing solutions are more effective at reducing pain from insertion of intravenous catheters. Patient-perceived pain related to periorbital injections of local anaesthesia is reduced when the anaesthetic is diluted with benzyl alcohol-preserved saline compared with other dilution or buffering options. Pain is similarly reduced for periocular botulinum toxin injections reconstituted with preserved saline compared with unpreserved saline. Benzyl-alcohol preserved-saline is inexpensive but costlier than unpreserved-saline, with minimal reported complications, particularly with periocular administration, and offers the opportunity to improve the patient experience.
\end{abstract}

Eye (2022) 36:1546-1552; https://doi.org/10.1038/s41433-021-01925-z

\section{INTRODUCTION}

Benzyl alcohol is an aromatic alcohol, a naturally occurring clear liquid produced by plants such as jasmine, and features in a wide variety of household products, cosmetics and foods, including fruits and teas. In healthcare it is used to treat head lice, as a local anaesthetic and as a preservative for a variety of drugs [1-6]. It has inherent anaesthetic and bacteriostatic properties and has been administered locally and systemically to reduce pain associated with needles or drug administration, and to reduce risk of intravenous catheter-related infections [7-11]. This article explores the current evidence for the use of benzyl alcohol-preserved saline in the context of periocular injections, specifically for reconstituting botulinum toxin and local anaesthesia.

\section{METHODS \\ A literature search using PubMed and Medline was carried out with search terms 'bacteriostatic saline' 'benzyl alcohol saline', 'benzyl alcohol sodium chloride' and 'preserved saline'. Relevant articles and their bibliographies were reviewed to identify further pertinent sources. \\ Benzyl alcohol for anaesthesia \\ Peri-procedural pain is a great source of anxiety for patients and can negatively impact patient engagement with healthcare}

services; minimising such pain is a source of great interest to patients and clinicians alike. As an ester precursor, benzyl alcohol has inherent anaesthetic properties [8]. It is available as a $0.9 \%$ solution with $0.9 \%$ sodium chloride (preserved saline, PS) [12]. PS has been explored as an alternative local anaesthetic option for a variety of indications. These include pain associated with: venipuncture [13], insertion of intravenous catheters [14-17], subcutaneous drug administration [18], intravenous drug administration [10], intra-articular injections [19], cervical dilatation for suction and curettage [20], periocular surgery [21-24] and periocular botulinum toxin A injections [25-28].

Wightman and Vaughan [29] injected 6 different solutions intradermally in the back of 20 volunteers' hands in a singleblinded study [29]. Solutions injected were: unpreserved saline (normal saline, NS), PS, $0.5 \%$ lidocaine, $0.5 \%$ lidocaine with $0.1 \%$ methylparaben, $1 \%$ lidocaine and $1 \%$ procaine. PS resulted in significantly lower pain scores than all other solutions, with $85 \%$ reporting 'no discomfort'; no more than 15\% reported 'no discomfort' with any other solution. Good to excellent anaesthesia was reported for the first $2 \mathrm{~m}$, but became inadequate after 2-3 mins for PS, whereas lidocaine-containing solutions retained satisfactory anaesthesia for $20 \mathrm{~m}$.

Wilson and Martin [30] explored both injection-associated pain, and level of anaesthetic efficacy in a double blinded, randomised controlled trial comparing NS to PS with 1:100,000 epinephrine or

${ }^{1}$ Corneoplastic Unit, Queen Victoria Hospital, East Grinstead, England. ${ }^{凶}$ email: samanthahunt2@nhs.net 
buffered 1\% lidocaine with 1:100,000 epinephrine (BL1E). Injections were delivered on the volar aspect of the forearm. A visual analogue scale was used to evaluate pain of pinprick at baseline, $5,15,30$ and 45 mins after administration, as well as the pain from the injection itself. Injection with PS with 1:100,000 epinephrine was significantly less painful than either NS or BL1E (48\% and $42 \%$ less painful, respectively). However, the anaesthesia produced was less than that of BL1E throughout. The authors concluded that PS with 1:100,000 epinephrine offers prolonged cutaneous local anaesthesia with less pain than BL1E and offers a good alternative option, especially if patients are allergic to lidocaine. Joliffe and Sinclair [31] report using PS successfully for an excision biopsy of an atypical forearm naevus in a patient with a previous adverse reaction to local anaesthetic. Lugo-Janer et al. [32] undertook a multi-phase study with phase one very similar to Wilson and Martin [30], involving volar forearm injections and pinprick testing up to $60 \mathrm{~m}$ post-injection [32]. They compared $1 \%$ lidocaine with 1:100,000 epinephrine (L1E), BL1E, PS with 1:300,000 epinephrine, and PS with L1E. PS-containing solutions resulted in statistically significantly lower injection-associated pain scores than all others, and all solutions offered adequate anaesthesia to a minimum of $30 \mathrm{~m}$ post-injection. Results were similar in phases 2 and 3 which evaluated the same anaesthetic solutions prior to superficial surgeries such as shave biopsies, and deep punch biopsies, respectively. The addition of sodium bicarbonate to lidocaine solutions raises the $\mathrm{pH}$ level and it is this alteration that is postulated to decrease pain levels [33]. The pH levels of PScontaining solutions in this study were 5.3 without lidocaine, 4.2 with lidocaine, both lower than that of BL1E at 7.4 and thus the authors concluded that the reduced injection-associated pain levels with PS-containing solutions is independent of $\mathrm{pH}$ level and related to the intrinsic anaesthetic effect of the benzyl alcohol [32]. Neither of these studies included PS without epinephrine. It is noteworthy that epinephrine has previously been shown to increase injection-associated pain levels when administered in the periocular region [22]. It appears that the addition of epinephrine prolongs the effect of PS-induced anaesthesia. It remains unknown, however whether injection-associated pain differs between plain PS and PS combined with epinephrine.

A 2014 meta-analysis incorporating 13 randomised controlled trials evaluating reduction of pain prior to intravenous catheter insertion concluded that lidocaine-containing solutions offer superior analgesia compared with PS alone when administered intradermally prior to insertion of an intravenous catheter [15]. However, the study did not consider injection-associated pain, which has been found to be similar, or less painful for PS than lidocaine-containing solutions [9, 13, 14, 16, 17, 34]. Furthermore, at the time of the meta-analysis, lidocaine was in critically shortsupply, and the authors highlighted that given PS does offer some anaesthesia, it may be more cost-effective than lidocaine-solutions when considering logistics of drug availability [15].

Frenken et al. [18] evaluated the impact of dilution of the paininducing vehicle used in subcutaneous injection of human recombinant erythropoietin with $0.54 \%$ PS or with $3 \%$ saline, that latter of which was designed to create an iso-osmotic solution. They undertook a randomised, double-blind, placebo-controlled study in healthy volunteers. Injection-associated pain was statistically significantly lower in the PS group than that of the undiluted vehicle, or the $3 \%$ saline-diluted vehicle, and was similar to that associated with NS, which acted as placebo. The effective osmotic value of the PS-diluted solution fell between those of the undiluted vehicle and iso-osmotic $3 \%$ saline-diluted solution and thus the authors believed the lower pain levels of the PS group were independent of osmotic pressure and related to the intrinsic local anaesthetic properties of PS. St Peter et al. [35] conducted a triple blinded, placebo controlled, randomised controlled trial on haemodialysis patients already requiring subcutaneous administration of erythropoietin. On two separate occasions, patients received a NS placebo injection in one arm and their usual dose of erythropoietin diluted with either NS or PS. The following visit, they again received NS placebo in one arm and their usual erythropoietin dose in other, this time diluted with whichever form of saline they had not received previously. Pain levels were assessed at $0,10,15$ and $30 \mathrm{~m}$. Maximal pain scores were reported at $5 \mathrm{~m}$ for PS and $10 \mathrm{~m}$ for NS, with lower scores in the PS injections reaching statistical significance. Interestingly, the authors also identified that patients with diabetes mellitus experienced significantly more pain than those without.

Zakria et al. [36] compared injection-associated pain in patients receiving intralesional triamcinolone for dermatological pathologies, where solutions contained either L1E or PS. This was a randomised, double-blinded, controlled trial but did not specify ratios of triamcinolone to local anaesthetic or PS, which was determined according to the clinical indication. Solutions containing PS were significantly less painful than those containing local anaesthetic (2/10 compared with $5 / 10$ on a visual analogue scale) and the authors recommended cessation of practice utilising local anaesthesia for pain amelioration in these patients.

Kruse et al. [19] undertook a double-blinded randomised controlled trial to compare use of PS with BL1 solutions as anaesthetic agents injected into skin, subcutaneous, and intramuscular tissues prior to ultrasound-guided intra-articular hip injection. No difference was found in pain during anaesthetic injection or the subsequent intra-articular injections between PS and BL1. However, Storey et al. [37] compared use of PS or NS to dilute contrast injected intra-articularly into the shoulder prior to MRI arthrography, and found statistically higher pain levels in those receiving PS throughout the $48 \mathrm{~h}$ follow-up period. This was a double blinded, randomised controlled trial, and all patients received subcutaneous BL1 prior to intra-articular injection. A notable limitation of the study was the failure to standardise, or record, other methods of analgesia used concurrently by the patients.

A double-blinded randomised controlled trial comparing PS and BL1, administered prior to cervical dilatation for suction and curettage in the submucosal paracervical region for cervical anaesthesia, found no difference in pain scores between the two groups for up to $30 \mathrm{~m}$ post-injection [20]. However, a 2009 double blinded, randomised controlled trial compared paracervical blocks with $1 \%$ chloroprocaine with PS as analgesia for elective termination of pregnancy [38]. They reported statistically higher pain scores in those receiving PS during administration of the block, aspiration of uterine contents and retrospective recollection of procedural pain. Chloroprocaine was chosen due to its rapid onset and short duration, and $3 \mathrm{~m}$ was allowed to pass between injection and the procedure. They did not evaluate pain associated with cervical dilatation since their patients did not require this.

PS has been shown to reduce pain associated with intravenous administration of propofol. Minogue and Sun [10] evaluated techniques to reduce pain associated with intravenous administration of propofol. They compared pre-injection of NS or PS, with $2 \%$ lidocaine-diluted propofol in a double-blinded randomised controlled trial. Both PS and lidocaine-diluted propofol groups reported statistically significantly lower pain scores for injection of propofol compared with NS, but no difference was found between these two groups. However, $20.5 \%$ of patients complained of mild pain during injection of the PS in comparison to $2.4 \%$ in the lidocaine group.

A 2019 meta-analysis explored various techniques and modifications that have been trialled to reduce pain associated with local anaesthesia injections specifically to eyelids and the periocular region. A wide variety of techniques have been adopted with supporting evidence available for the following: solution modification (buffering, dilution, warming), skin cooling with ice, tactile distraction with vibration, slower rate of injection 
[21]. The meta-analysis included one study by Yuen and Dolman [23] incorporating PS for anaesthesia in the periocular region in a double-blinded randomised controlled trial comparing injectionassociated pain, and efficacy of anaesthesia for eyelid surgery [23]. They compared $2 \%$ lidocaine with 1:100,000 epinephrine (L2E), buffered $2 \%$ lidocaine with 1:100,000 epinephrine (BL2E), and L2E diluted 1:9 with PS. Patients underwent identical bilateral surgery, receiving different anaesthetic solutions to each eyelid. Injectionassociated pain, and operation-associated pain was reported using a 5-point pain scale. Lidocaine diluted with PS received significantly lower pain scores than either L2E or BL2E. Mean intraoperative pain scores were $<1$ for all forms of anaesthesia, and no significant differences were found in intraoperative pain, though the actual reported pain scores are not stipulated. The authors conclude that PS-diluted L2E offers adequate intraoperative anaesthesia for eyelid surgery with less pain during injection than either L2E or BL2E. In a letter response to the metaanalysis, Dolman and Yuen [24] highlighted their observation and opinion that PS had an additional value over and above the diluting effect of un-preserved saline in being the important additional factor in reducing pain of injection. They also pointed out that they currently used a modified 1:1 ratio of L2E:PS solution as they perceive it has a longer lasting, strong anaesthetic effect but still benefits from minimal injection-related pain.

\section{Preserved saline for periocular botulinum toxin injections}

First introduced for management of pathologies such as strabismus and blepharospasm, botulinum toxin A injections were adopted for cosmetic treatments in the mid-1980s [39, 40]. Recently, there has been a year-on-year increase in demand for cosmetic botulinum toxin A injections, with 3.19 billion USD spent in North America in 2019, although therapeutic indications still dominate the market [41, 42]. The current Covid-19 pandemic dramatically affected how people interact, as well as available funds for more 'frivolous' spending; 'Zoom dysmorphia' is a proposed explanation for demand for cosmetic botulinum toxin injections remaining stable throughout this period, and more invasive cosmetic surgical procedures such as rhinoplasty and blepharoplasty increasing [43].

In 2002, Alam et al. [25] reported their work on injectionassociated pain levels in individuals receiving botulinum toxin $A$ injections to the upper face for dynamic facial lines [25]. Numbers were small; in the first part of their research, they asked 20 patients who had previously received injections reconstituted with NS to estimate the percentage that their next injection, reconstituted with PS, was more or less painful than previously. A total of 18 of 20 patients reported an average of $55 \%$ less pain. They then completed a prospective double-blinded randomised controlled trial in which 15 different patients received injections to one side of the face reconstituted with NS, the other with PS. All patients reported less pain with PS, averaging 54\% less painful. Patient-reported effectiveness of the injections was recorded at follow-up and did not suggest decreased effectiveness. These findings were replicated by Allen and Goldberg [28] who similarly injected abobotulinum toxin A reconstituted with either NS or PS to each side of the face in a double-blinded randomised controlled trial, with patients reporting $60 \%$ less pain with PS than NS, using a visual analogue scale. Patients were examined at 2 weeks following injection with no clinical or patient-reported evidence of altered effectiveness between the two solutions. Zidan et al. [44] undertook a double-blinded, randomised controlled trial administering botulinum toxin injections following a standardised protocol for patients with chronic migraine. This involved injections to corrugators, procerus frontalis, temporalis, occipitalis, cervical paraspinal and trapezius muscles. 68 consecutive patients were enroled and randomised to receiving botulinum toxin reconstituted with either PS or NS, and kept headache diaries for 1 week following injection. Patients reported statistically significantly lower injection-associated pain scores in the PS group, with no difference in post-injection headache rates.

Kwiat et al. [26] undertook similar work for patients receiving botulinum toxin $A$ injections for blepharospasm. 20 patients all received injections reconstituted with NS to one eye and PS to the other in a double-blinded randomised controlled trial. Of the 20 patients, 19 preferred PS over NS. Using a 10-point verbal pain scale, $89 \%$ and $63 \%$ of patients reported pain of at least 3 points lower and 4 points lower, respectively with PS compared with NS. No difference was found in effectiveness of the injections via patient-reported or clinical examination findings at follow-up.

Van Laborde et al. [27] replicated these findings with injections of botulinum toxin $B$ injected to the upper face for dynamic facial lines. A double-blinded randomised controlled trial of 15 patients identified $32 \%$ reduced pain levels for injections reconstituted with PS than NS which was statistically significant. No difference in clinical effectiveness was reported by patients or identified by clinicians.

Sarifakioglu and Sarifakioglu [45] compared pain scores associated with botulinum toxin $A$ injections to 3 different sitesperiorbital, neck and axillae, with right sides receiving toxin reconstituted with PS and left sides toxin reconstituted with NS. Although they report the study as a single blinded randomised controlled study, there does not appear to be any randomisation as patients received treatment to sites according to clinical signs. Furthermore, the authors report contacting patients at several points up to 6 months post-injection to establish treatment efficacy, but do not report their results. They did, however, report significantly lower injection-associated pain on the PS treatment side for all 3 injection sites.

In 2014, Huang et al. [46] reported a retrospective cohort study of 88 patients, undergoing 300 injections of onabotulinum toxin A reconstituted with PS to the central eyelid or eyebrow [7]. Of these, 31 patients (50 injections) were for cosmetic purposes. Concentrations injected were either 2.5 units $/ 0.1 \mathrm{ml}$ or 5 units $/ 0.1 \mathrm{ml}$. Outcome measures were rates of documented complications including diplopia, ptosis, lagophthalmos, blurred vision and local effects of pain, bruising, bleeding or swelling. Overall complication rates were low: $4 \%$ diplopia, $1 \%$ ptosis, $1 \%$ lagophthalmos, $1 \%$ blurred vision, 3.3\% local effects. Patients who reported 1 complication were more likely to report another complication. However, complications rates were statistically significantly higher in those receiving treatment for functional indications rather than cosmetic, and in those receiving the higher concentration. Unfortunately, a detailed breakdown was not available to compare the differences in local effect complications to the different concentrations. This was a retrospective review and did not include a discussion regarding decision-making processes for which patients received each concentration of toxin. However, those receiving the higher concentration were noted to receive higher doses overall, more injections, and injections in more aggressive locations. It may not be appropriate, therefore, to compare the rates of complications between these groups, since they may represent two distinct patient groups.

\section{Bacteriostatic properties of PS}

Rein and Mandell [47] demonstrated the bacteriostatic effect of PS in vitro. They incubated 7 different species of bacteria in 4 different solutions-NS, PS, saline with parabens and lactated Ringer's solution. After $60 \mathrm{~m}$ of room-temperature incubation, millifilters were used to remove the vehicle solution and then these were cultured. PS showed the lowest percentage of surviving bacteria across all 7 species, with $0 \%$ surviving the 60 min incubation period of Haemophilus Influenzae, Streptococcus Pneumoniae and Streptococcus Viridans.

Evidence suggests that the bacteriostatic effect of benzyl alcohol in PS reduces the risk of colonisation and infection over 
NS. Shenep et al. [48] undertook similar in vitro research to examine anti-microbial properties of heparin-containing solutions. They incubated 6 different organisms in heparin, heparin with PS, heparin with parabens, $70 \%$ ethanol, and NS at 35 degrees centigrade, taking samples for culture after 2, 4 and $24 \mathrm{~h}$. Ethanol was the most effective antimicrobial, eliminating most organisms within $4 \mathrm{~h}$. Solutions containing PS or parabens were equally effective, eliminating most organisms within $24 \mathrm{~h}$, and heparin alone or NS were ineffective. They also reviewed infection control records for central venous catheter infections in their paediatric population over a 26-year period. They identified 2 spikes in outpatient-related central venous catheter infections which were attributable to the withdrawal, or dramatic reduction in concentration of preservative in the heparin-lock solutions used to prevent infections and which resolved upon re-introduction of PScontaining solutions.

Wiernikowski et al. [7] also reported a possible benefit to using PS rather than NS for flushing right atrial catheters in preventing line infections. In a double-blinded randomised controlled trial, children with malignant diseases had their tunnelled right atrial catheter lines flushed weekly with either NS or PS. Weekly blood cultures drawn from the indwelling catheter were used alongside clinical 'events' of sepsis to establish rates of line infection. Event rates suggested a trend in favour of the use of PS but did not reach statistical significance over the 6 month trial period. However, time to first infection was significantly longer in the PS group and results would have been statistically significant had the trial ceased at 3 months rather than 6 months duration. The authors acknowledged the possibility of a seasonal effect-the first event in the PS group occurred 17 weeks into the trial, at the beginning of the summer, and the authors felt that children might be at increased risk of infections by playing outside or swimming more during the warmer months, though no evidence was provided supporting this theory. Drawing on the overall delayed time to first event, the authors concluded that flushing the lines with PS is beneficial in reducing line-colonisation and associated infections.

Luther et al. undertook a large retrospective cohort study evaluating infection rates in all patients receiving intralesional triamcinolone over a 1 -year period. $64 \%$ of triamcinolone injections were reconstituted with PS. 11 of 4370 patients had evidence of an infection within 30 days of injection, 4 in the PS group, 7 in the NS group. All 4 patients in the PS group had received an injection into an 'inflamed cyst' compared with 3 of the 7 in the NS group. Although there was a trend to lower infection rates in the PS group, this did not reach statistical significance.

In 2009, Pham et al. [49] reported a large retrospective cohort study of individuals receiving botulinum toxin $A$ injections reconstituted with PS to the periocular/facial region for a variety of indications, including blepharospasm, hemifacial spasm, aberrant nerve regeneration and cosmesis. No patient received pre-injection skin antisepsis with isopropyl alcohol and needles were not changed between injections where patients had multisite injections. 142 patients received 11, 627 individual injections, with no reported injection site infections. The authors acknowledge that very low infection rates mean proving non-inferiority is challenging, but their data suggests very low infection rates with botulinum toxin $A$ injections even without prior skin antisepsis. Furthermore, the authors note that changes in cutaneous microcirculation with topical antisepsis thicken the skin, and they highlight anecdotal reports of increased pain when injections are given following its usage. Given the low rate of infections with injections of botulinum toxin $A$ to the periocular/facial regions when it is reconstituted with PS, they believe that pre-injection isopropyl alcohol skin antisepsis is unnecessary and may negatively impact patient experience.

\section{Other complications of benzyl alcohol}

Concerns exist regarding systemic toxicity of benzyl alcohol. However, the general public are regularly exposed to benzyl alcohol via food, and the European Medicines Agency [2] have set an oral Acceptable Daily Intake limit of $5 \mathrm{mg} / \mathrm{kg}$ for individuals over 4 weeks of age. Severe toxicity, possibly contributing to the death of newborn pre-term neonates has occurred with the systemic administration of PS, and its use is now contraindicated in this population $[2,6,50,51]$. Concerns also exist around intrathecal administration of PS, with reported neurological toxicity, especially where concentrations of at least $0.9 \%$ benzyl alcohol are used [52-55]. Although Reynolds [56] raised concerns that inhalation of PS via nebulisers can induce bronchitis, the same author showed that inhalation of either PS or NS can induce bronchitis in healthy volunteers, and further research is required to delineate this risk further [57].

The general public are also frequently exposed to benzyl alcohol via cosmetics and household products [1-4]. The potential for benzyl alcohol to result in contact dermatitis was recognised in 1975 , and it now features on allergy patch testing lists $[4,58,59]$. Clinicians must be aware of the potential for patient-reported allergies to cosmetics or substances such as balsam of Peru that may represent an allergy to benzyl alcohol [60]. Amado and Jacob [60] report 2 patients with intractable eyelid dermatitis found to be related to PS-reconstituted botulinum toxin injections and resolved when future injections were reconstituted with NS. PS should be avoided if any concerns regarding allergy exist. Currently, no evidence exists on whether repeated exposure via such injections might increase the risk of subsequent allergy.

\section{Practical considerations when using PS}

PS is FDA approved [12]. Although costs of drugs may change according to local contracts, suppliers and stock availabilities, PS is currently advertised at $\$ 4.95$ for a $20 \mathrm{ml}$ multi-use vial [61], or $£ 16.50$ (\$22.17 USD) for a pack of $10 \times 5 \mathrm{ml}$ ampoules [62]. Using PS is therefore more expensive than NS for reconstituting botulinum toxin, and more expensive than using either undiluted lidocaine/bupivacaine or lidocaine/bupivacaine diluted with NS. However, it is less expensive than lidocaine/bupivacaine diluted with sodium bicarbonate.

Ganter-Ritz et al. [14] found PS to be the most cost-effective option of $1 \%$ lidocaine, BL1, and PS, but Oman et al. [15] rightly highlight the complexities of definitive cost-analysis based on a variety of variable factors including: single vs multi-use vials of products, number of draw up needles needed, refrigeration requirements, product stability. Furthermore, local contracts between units and their suppliers may also impact the cost of the products. However, the cost difference between products is not substantive, particularly when considered in the context of the total cost for the surgical or medical procedure that local anaesthesia is required for in order to minimise patientperceived pain.

Storage and stability of reconstituted botulinum toxin products has been a matter of some interest in the literature [39, 63]. Allergan, Inc [64] manufacture BOTOX (onabotulinum toxin A) and only recommend reconstitution with NS, after which it should be refrigerated $\left(2-8{ }^{\circ} \mathrm{C}\right)$ and used within $4 \mathrm{~h}$. However, many clinicians use a reconstituted botulinum toxin $A$ solution well beyond this recommended time-frame, with conflicting evidence regarding altered efficacy with delayed use, some studies suggesting efficacy up to 6 weeks after reconstitution with appropriate storage $[63,65]$. The effect of PS rather than NS on product stability and sterility has also been considered; Alam et al. [65] conducted a study simulating multiple uses of reconstituted, stored botulinum toxin A over a period of 7 weeks and found no microbiological evidence of contamination. However, they did not explore the efficacy of the product after storage in this manner and use of botulinum toxin beyond the recommended time frame 
is unlicensed. Given the expense of botulinum toxin A injections and availability in multi-use vials, many institutions run dedicated botulinum toxin clinics where the number of units required can be predicted; this minimises waste, maximises cost-efficiency and eliminates the need for storage of reconstituted product.

\section{Areas for further research}

Buffering lidocaine-containing solutions with sodium bicarbonate decreases injection-associated pain [21]. No studies have explored the addition of sodium bicarbonate to PS to evaluate the impact this has on injection-associated pain. However, with very low pain scores for PS injections, proving superiority of a buffered PS solution may be challenging, and the addition of the sodium bicarbonate would have logistical implications such as increased cost and time for preparation, and reduced product stability, and may therefore not be beneficial.

Although reconstitution concentrations for botulinum toxin $A$ are fairly standardised, a variety of dilution ratios of lidocaine have been used both with NS and PS [21, 23, 24, 63, 64]. There are as yet no comparative studies to inform clinicians about optimal ratios for minimal injection pain and maximal anaesthetic effect. Optimal dilution ratios may vary depending on the clinical indication, and thus the duration of anaesthesia required, as well as patient factors such as pain tolerance. In addition, the effect of adding epinephrine to PS appears to prolong the duration of anaesthesia, but any impact on injection-associated pain has yet to be established.

\section{CONCLUSION}

Evidence for the efficacy and value of PS compared with amide anaesthetics is mixed when used for intra-articular or cervical injections and as pre-cannulation anaesthesia. However, there is now a wealth of evidence that injection-associated pain is decreased for patients receiving periocular botulinum toxin or local anaesthetic injections with use of PS for reconstitution or dilution of these solutions.

Botulinum toxin injections reconstituted with PS are equally efficacious as those reconstituted with NS. It is unclear how long PS-reconstituted product can be stored for and retain efficacy, but contamination does not appear to be an issue up to 7 weeks after reconstitution, even with multiple vial accesses. Complications rates appear to be very low, with no evidence to suggest these are higher than when NS is used to reconstitute botulinum toxin. Similarly, infection rates with periocular botulinum toxin injections are very low even without prior skin antisepsis, though clinicians should ensure they adhere to local infection control policies or challenge these using available evidence.

Multiple studies have compared injection-associated pain levels for facial and periocular botulinum toxin injections when administered for both cosmetic and therapeutic indications. Patients receiving treatment for therapeutic indications reportedly experience higher complications from these injections than those undergoing cosmetic treatment but patient-reported injectionassociated pain levels are consistently lower when botulinum toxin is reconstituted with PS, with $32-60 \%$ reduction in pain levels compared with NS.

Unmodified PS offers almost painless but short-lived (2-3 m) anaesthesia, which may be suitable for some indications, including for periocular botulinum toxin injections, and is a cheap and convenient option. However, PS can also be combined with lidocaine and/or epinephrine to increase the duration of anaesthesia whilst still reducing overall injection-associated pain compared with various lidocaine-containing solutions for larger periocular surgical procedures. Yuen and Dolman [23] reported lower injection-associated pain levels when L2E was diluted with 1:9 PS, with very good intraoperative anaesthesia for periocular surgery. They have since modified their dilution ratio to $1: 1$ but have not reported outcome data evaluating the effect of this change, and it is therefore unclear what the optimal dilution ratio is, and whether this depends on the clinical indication. Furthermore, it is recognised that the addition of epinephrine to lidocaine solutions increases injection-associated pain, but there are no studies comparing injection-associated pain between PSdiluted lidocaine and those that also contain epinephrine.

There is evidence to support the bacteriostatic properties of PS in vivo, with studies reporting a positive impact on indwelling-line associated infections. Pham et al. report no infections in more than 11,000 periocular botulinum toxin injections, where the toxin was reconstituted with PS despite withholding isopropyl alcohol skin antisepsis. However, there are no direct comparisons in infection rates between botulinum toxin reconstituted with NS and PS, and with such low infection rates with this procedure, statistically proving superiority requires prohibitively large cohorts. Furthermore, there is no discussion in the literature of infection rates after periocular surgery where lidocaine-containing solutions have been diluted with PS. Although risks of systemic toxicity with use of periocular PS is incredibly small, there is a reasonable possibility of benzyl alcohol allergy; manifestation via a localised dermatitis has been reported following periocular botulinum toxin injections that resolved when further injections were reconstituted with NS. As with the administration of any drug, clinicians must always be prepared to manage anaphylaxis, though this has not been reported with periocular administration of PS. Use of PS is more expensive than NS, or undiluted lidocaine-containing anaesthetics, but would likely represent a cost-saving for clinicians currently diluting lidocaine-containing solutions with sodium bicarbonate buffer solution, and would also result in significantly reduced injection-associated pain for the patient.

\section{REFERENCES}

1. Shmunes E. Allergic dermatitis to Benzyl alcohol in an injectable solution. Arch Dermatol. 1984;120:1200-1.

2. European Medicines Agency. Benzyl alcohol and benzoic acid group used as excipients. 2017:16. https://www.ema.europa.eu/en/documents/report/benzylalcohol-benzoic-acid-group-used-excipients-report-published-support-questionsanswers-benzyl/chmp/508188/2013-t_en.pdf. Accessed 26 Aug 2021.

3. Curry EJ, Warshaw EM. Benzyl alcohol allergy: importance of patch testing with personal products. Dermatitis.2005;16:203-8.

4. London Allergy and Immunology Centre. Patch Testing, Patch Tests \& Chemical Allergy Tests [Internet]. London Allergy and Immunology Centre @ Harley Street Medical Centre UK (Private Allergy and Skin specialists). [cited 2021 Aug 26]. Available from: https://www.allergycliniclondon.co.uk/allergy-tests/patch-testing/.

5. Meinking TL, Villar ME, Vicaria M, Eyerdam DH, Paquet D, Mertz-Rivera K, et al. The clinical trials supporting Benzyl alcohol lotion 5\% (UlesfiaTM): a safe and effective topical treatment for head lice (Pediculosis Humanus Capitis). Pediatr Dermatol. 2010;27:19-24.

6. Nair B. Final report on the safety assessment of Benzyl alcohol, Benzoic acid, and sodium Benzoate. Int J Toxicol. 2001;20:23-50.

7. Wiernikowski JT, Elder-Thornley D, Dawson S, Rothney M, Smith S. Bacterial colonization of tunneled right atrial catheters in pediatric oncology: a comparison of sterile saline and bacteriostatic saline flush solutions. Am J Pediatr Hematol Oncol. 1991;13:137-40.

8. Macht DI, Leach HP. Concerning the antipyretic properties of Benzyl Benzoate. J Pharmacol Exp Ther. 1929;35:281-96.

9. Fein JA, Boardman CR, Stevenson S, Selbst SM. Saline benzyl alcohol intradermal anesthesia intravenous line Place Child: Pediatr Emerg Care. 1998;14:119-22.

10. Minogue SC, Sun DA. Bacteriostatic saline containing benzyl alcohol decreases the pain associated with the injection of propofol. Anesth Analg. 2005;100:683-6.

11. McCarthy TJ, Ferreira J-H. Attempted measurement of the activity of selected preservative combinations. J Clin Pharm Ther. 1990;15:123-9.

12. Drugs@FDA: FDA-Approved Drugs [Internet]. [cited 2021 Sep 5]. Available from: https://www.accessdata.fda.gov/scripts/cder/daf/index.cfm?event=overview. process\&AppINo $=088911$.

13. Patterson $P$, Hussa $A A$, Fedele KA, Vegh $G L$, Hackman CM. Comparison of 4 analgesic agents for venipuncture. AANA J. 2000;68:43-51.

14. Ganter-Ritz V, Speroni KG, Atherton M. A randomized double-blind study comparing intradermal anesthetic tolerability, efficacy, and cost-effectiveness of 
lidocaine, buffered lidocaine, and bacteriostatic normal saline for peripheral intravenous insertion. J Infus Nurs. 2012;35:93-9.

15. Oman KS, Fink R, Kleiner C, Makic MBF, Wenger B, Hoffecker L, et al. Intradermal lidocaine or bacteriostatic normal saline to decrease pain before intravenous catheter insertion: a meta-analysis. J Perianesth Nurs. 2014;29:367-76.

16. Deguzman ZC, O'Mara SK, Sulo S, Haines T, Blackburn L, Corazza J. Bacteriostatic normal saline compared with buffered $1 \%$ lidocaine when injected intradermally as a local anesthetic to reduce pain during intravenous catheter insertion. J Perianesth Nurs. 2012;27:399-407.

17. McNelis KA. Intradermal bacteriostatic $0.9 \%$ sodium chloride containing the preservative benzyl alcohol compared with intradermal lidocaine hydrochloride $1 \%$ for attenuation of intravenous cannulation pain. AANA J. 1998;66:583-5.

18. Frenken LAM, van Lier HJJ, Koene RAP. Analysis of the efficacy of measures to reduce pain after subcutaneous administration of epoetin alfa. Nephrol Dial Transplant [Internet]. 1994 [cited 2021 Sep 30]; Available from: https:// academic.oup.com/ndt/article/9/9/1295/1848848/Analysis-of-the-efficacy-ofmeasures-to-reduce.

19. Kruse RC, Kindle BJ, Wisniewski S, Presley J, Smith J, Sellon JL. Local anesthesia prior to ultrasound-guided hip joint injections: a double-blind randomized controlled trial of bacteriostatic saline versus buffered lidocaine. PMR.2021;13:811-8.

20. Miller L, Jensen MP, Stenchever MA. A double-blind randomized comparison of lidocaine and saline for cervical anesthesia. Obstet Gynecol. 1996;87:600-4.

21. Gostimir M, Hussain A. A systematic review and meta-analysis of methods for reducing local anesthetic injection pain among patients undergoing periocular surgery. Ophthal Plast Reconstr Surg. 2019;35:113-25.

22. Han J, Nah SK, Lee SY, Kim CY, Yoon JS, Jang SY. A prospective, comparative study of the pain of local anesthesia using $2 \%$ lidocaine, $2 \%$ lidocaine with epinephrine, and $2 \%$ lidocaine with epinephrine-bupivicaine mixture for eyelid surgery. Ophthal Plast Reconstr Surg. 2017;33:132-5.

23. Yuen VH, Dolman PJ. Comparison of three modified lidocaine solutions for use in eyelid anesthesia. Ophthal Plast Reconstr Surg. 1999;15:143-7.

24. Dolman PJ, Yuen VH. Re: 'a systematic review and meta-analysis of methods for reducing local anesthetic injection pain among patients undergoing periocular surgery'. Ophthal Plast Reconstr Surg. 2019;35:412-3.

25. Alam M, Dover JS, Arndt KA. Pain associated with injection of botulinum a exotoxin reconstituted using isotonic sodium chloride with and without preservative: a double-blind, randomized controlled trial. Arch Dermatol. 2002;138:510-4.

26. Kwiat DM, Bersani TA, Bersani A. Increased patient comfort utilizing botulinum toxin type a reconstituted with preserved versus nonpreserved saline. Ophthal Plast Reconstr Surg. 2004;20:186-9.

27. van Laborde S, Dover JS, Moore M, Stewart B, Arndt KA, Alam M. Reduction in injection pain with botulinum toxin type $B$ further diluted using saline with preservative: a double-blind, randomized controlled trial. J Am Acad Dermatol. 2003;48:875-7.

28. Allen SB, Goldenberg NA. Pain difference associated with injection of abobotulinumtoxina reconstituted with preserved saline and preservative-free saline: a prospective, randomized, side-by-side, double-blind study. Dermatol Surg. 2012;38:867-70.

29. Wightman MA, Vaughan RW. Comparison of compounds used for intradermal anesthesia. Anesthesiology.1976;45:687-9.

30. Wilson L, Martin S. Benzyl alcohol as an alternative local anesthetic. Ann Emerg Med. 1999;33:495-9.

31. Jolliffe VML, Sinclair RD. Excision biopsy in a patient with suspected local anaesthetic allergy: use of $0.9 \%$ saline with benzyl alcohol as local anaesthesia: Use of $0.9 \%$ saline with benzyl alcohol as local anaesthesia in an allergic patient. Clin Exp Dermatol. 2012;37:862-4.

32. Lugo-Janer G, Padial M, Sánchez JL. Less painful alternatives for local anesthesia. J Dermatol Surg Oncol. 1993;19:237-40.

33. McKay W, Morris R, Mushlin P. Sodium bicarbonate attenuates pain on skin infiltration with lidocaine, with or without epinephrine. Anesth Analg. 1987;66:572-4.

34. Burgher SW, McGuirk TD. Subcutaneous buffered lidocaine for intravenous cannulation: is there a role in emergency medicine? Acad Emerg Med. 1998;5: 1057-63.

35. Peter W, Lewis $M$, Macres M. Pain comparison after subcutaneous administration of single-dose formulation versus multidose formulation of epogen in hemodialysis patients. Am J Kidney Dis. 1998;32:470-4.

36. Zakria D, Patrinely JR, Dewan AK, Albers SE, Wheless LE, Simmons AN, et al. Intralesional corticosteroid injections are less painful without local anesthetic: a double-blind, randomized controlled trial. J Dermatol Treat. 2021:7;1-4.

37. Storey TF, Gilbride G, Clifford K. Postprocedural pain in shoulder arthrography: differences between using preservative-free normal saline and normal saline with benzyl alcohol as an intraarticular contrast diluent. Am J Roentgenol. 2014;203:1059-62.

38. Glantz JC, Shomento S. Comparison of paracervical block techniques during first trimester pregnancy termination. Int J Gynecol Obstet. 2001;72:171-8.
39. Carruthers A, Carruthers J. History of the cosmetic use of botulinum a exotoxin. Dermatol Surg. 1998;24:1168-71.

40. Clark RP, Berris CE. Botulinum toxin: a treatment for facial asymmetry caused by facial nerve paralysis. Plast Reconstr Surg. 2005;115:573-4.

41. Fortune Business Insights. Botulinum Toxin Market Size \& Share | Global Report [2020-2027] [Internet]. The global botulinum toxin market size was $\$ 4.83$ billion in 2019 \& is projected to reach $\$ 7.71$ billion by 2027 , exhibiting a CAGR of $7.5 \%$ in forecast period... 2020 [cited 2021 Aug 29]. Available from: https://www. fortunebusinessinsights.com/industry-reports/botulinum-toxin-market-100996.

42. Solutions TKISG American Academy of Facial Plastic and Reconstructive Surgery [Internet]. American Academy of Facial Plastic and Reconstructive Surgery. [cited 2021 Aug 29]. Available from: https://www.aafprs.org/.

43. Rice SM, Graber E, Kourosh AS. A pandemic of dysmorphia: "Zooming" into the perception of our appearance. Facial Plast Surg Aesthetic Med. 2020;22:401-2.

44. Zidan A, Hussaini S, Gibson S, Brooks G, Mejico L. Onabotulinumtoxin Type A reconstitution with preserved versus preservative-free saline in chronic migraine (B-RECON). A randomised, double-blind trial. Int J Clin Pract [Internet]. 2020 Sep [cited 2021 Sep 30];74. Available from: https://onlinelibrary.wiley.com/doi/ $10.1111 / \mathrm{ijcp} .13522$.

45. Sarifakioglu N, Sarifakioglu E. Evaluating effects of preservative-containing saline solution on pain perception during botulinum toxin Type-A injections at different locations: a prospective, single-blinded, randomized controlled trial. Aesthetic Plast Surg. 2005;29:113-5.

46. Huang L, Costin BR, Sakolsatayadorn N, Perry JD. Safety of onabotulinum toxin a injection to the central upper eyelid and eyebrow regions. Ophthal Plast Reconstr Surg. 2014;30:377-80.

47. Rein MF, Mandell GL. Bacterial killing by bacteriostatic saline solutions potential for diagnostic error. N. Engl J Med. 1973;289:794-5.

48. Shenep LE, Shenep MA, Cheatham W, Hoffman JM, Hale A, Williams BF, et al. Efficacy of intravascular catheter lock solutions containing preservatives in the prevention of microbial colonization. J Hosp Infect. 2011;79:317-22.

49. Pham T, Perry JD. Botulinum toxin type A injection without isopropyl alcohol antisepsis. Ophthal Plast Reconstr Surg. 2009;25:178-9.

50. American Academy of Paediatrics Committee on Fetus and Newborn. Benzyl Alcohol: Toxic Agent in Neonatal Units. Pediatrics. 1983;72:356-8.

51. Saito J, Nadatani N, Setoguchi M, Nakao M, Kimura $H$, Sameshima M, et al. Potentially harmful excipients in neonatal medications: a multicenter nationwide observational study in Japan. J Pharm Health Care Sci. 2021;7:23.

52. Hetherington NJ, Dooley MJ. Potential for patient harm from intrathecal administration of preserved solutions. Med J Aust. 2000;173:141-3.

53. Messinger YH, Maxa KL, Hennen EM, Gossai NP, Hoff DS. Intrathecal methotrexate containing the preservative benzyl alcohol erroneously administered in pediatric leukemia patients: clinical course and preventive process. J Pediatr Pharm Ther JPPT. 2020;25:328-31.

54. Craig DB, Habib GG. Flaccid paraparesis following obstetrical epidural anesthesia: possible role of benzyl alcohol. Anesth Analg. 1977;56:219-21.

55. Hahn AF, Feasby TE, Gilbert JJ. Paraparesis following intrathecal chemotherapy. Neurology.1983;33:1032-8.

56. Reynolds RD. Nebulizer bronchitis induced by bacteriostatic saline. JAMA J Am Med Assoc. 1990:264:35.

57. Reynolds RD, Smith RM. Nebulized bacteriostatic saline as a cause of bronchitis. J Fam Pr. 1995;40:35-40.

58. Fisher AA. Allergic paraben and benzyl alcohol hypersensitivity relationship of the "delayed" and "immediate" varieties. Contact Dermat. 1975;1:281-4.

59. Guin JD, Goodman J. Contact urticaria from benzyl alcohol presenting as intolerance to saline soaks: SHORT COMMUNICATION. Contact Dermat. 2001;45:182-3.

60. Amado A, Jacob SE. Letter: benzyl alcohol preserved saline used to dilute injectables poses a risk of contact dermatitis in fragrance-sensitive patients. Dermatol Surg. 2007;33:1396-7.

61. Mountainside Medical Equipment. Bacteriostatic Sodium Chloride $0.9 \%$ for Injection $20 \mathrm{ml}$ - Single Vial [Internet]. Mountainside Medical Equipment. [cited 2021 Sep 16]. Available from: https://www.mountainside-medical.com/products/ bacteriostatic-sodium-chloride-for-injection.

62. TOR-BAC Bacteriostatic Sodium Chloride Ampoules Solution [Internet]. Fox Pharma. [cited $2021 \mathrm{Sep}$ 30]. Available from: https://foxpharma.co.uk/product/ tor-bac-bacteriostatic-sodium-chloride-ampoules-solution/.

63. Klein AW. Dilution and storage of botulinum toxin. Dermatol Surg. 1998;24: $1179-80$.

64. Allergan, Inc. Highlights of Prescribing Information: BOTOX [Internet]. Allergan, Inc; 2021 [cited 2021 Sep 5]. Available from: https://media.allergan.com/actavis/ actavis/media/allergan-pdf-documents/product-prescribing/20190620-BOTOX100-and-200-Units-v3-0USPI1145-v2-0MG1145.pdf.

65. Alam M, Yoo SS, Wrone DA, White LE, Kim JYS. Sterility assessment of multiple use botulinum A exotoxin vials: a prospective simulation. J Am Acad Dermatol. 2006;55:272-5. 


\section{AUTHOR CONTRIBUTIONS}

$\mathrm{SH}$ : Reviewed all relevant literature, prepared the manuscript and text, instigated revisions where appropriate. RM: Supervised the limits of the review and article structure. Edited the manuscript and suggested revisions.

\section{COMPETING INTERESTS}

The authors declare no competing interests.

\section{ADDITIONAL INFORMATION}

Correspondence and requests for materials should be addressed to Samantha Vicki Hunt.

Reprints and permission information is available at http://www.nature.com/ reprints

Publisher's note Springer Nature remains neutral with regard to jurisdictional claims in published maps and institutional affiliations. 